BULLETIN (New Series) OF THE

AMERICAN MATHEMATICAL SOCIETY

Volume 45, Number 2, April 2008, Pages 315-319

S 0273-0979(07)01162-7

Article electronically published on August 8, 2007

Quadrangular algebras, by Richard M. Weiss, Mathematical Notes, vol. 46, Princeton University Press, 2006, x+131 pp., US\$45.00, ISBN 978-0-691-12460-5

One of the best-known and most beautiful parts of mathematics is the classification of the simple Lie groups over the complex numbers. This was achieved in the late 1880s by Wilhelm Killing, whose work was remodelled by Élie Cartan in 1894, and it is now referred to as the Killing-Cartan classification. A Lie group is called simple if it has no non-trivial, connected, normal subgroups, and the simple Lie groups over the complex numbers $\mathbf{C}$ are in bijective correspondence with the Dynkin diagrams in families A through G. Here are these well-known diagrams:
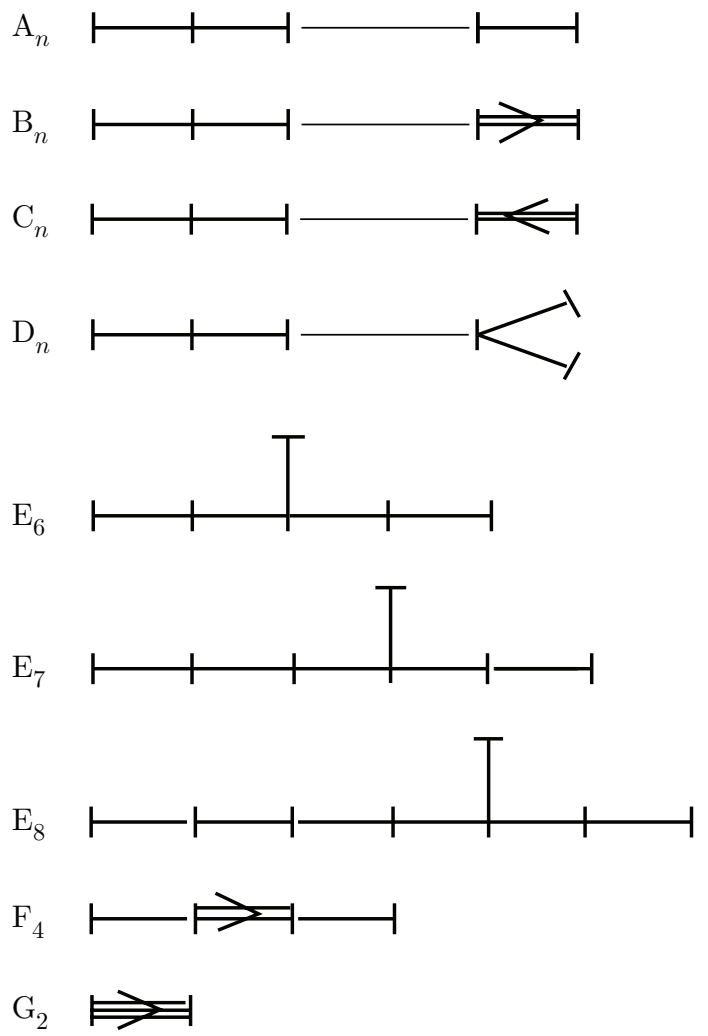

When one descends from the complex numbers to the real numbers, the classification of simple Lie groups becomes more complicated. Here is an example. The complex Lie group $\mathrm{SL}_{2}(\mathbf{C})$ is simple (its only normal subgroup is the group of diagonal matrices, which has order 2 and is not connected), and it has two "real" forms. One is obtained by restricting the entries of each 2-by-2 matrix to the real

2000 Mathematics Subject Classification. Primary 17D99; Secondary 20G15, 51B25.

(C) 2007 American Mathematical Society Reverts to public domain 28 years from publication 
numbers - this yields $\mathrm{SL}_{2}(\mathbf{R})$. The other is obtained as follows. Every complex 2by-2 matrix can be written as a linear combination of four matrices in the following way:

$$
\alpha\left[\begin{array}{ll}
1 & 0 \\
0 & 1
\end{array}\right]+\beta\left[\begin{array}{cc}
i & 0 \\
0 & -i
\end{array}\right]+\gamma\left[\begin{array}{cc}
0 & -1 \\
1 & 0
\end{array}\right]+\delta\left[\begin{array}{ll}
0 & i \\
i & 0
\end{array}\right] .
$$

The symbols $\alpha, \beta, \gamma, \delta$ represent complex numbers, and the resulting matrix will lie in the group $\mathrm{SL}_{2}(\mathbf{C})$ - in other words, it will have determinant 1 - when $\alpha^{2}+\beta^{2}+$ $\gamma^{2}+\delta^{2}=1$. Restricting $\alpha, \beta, \gamma, \delta$ to the real numbers yields a compact topological space, namely the 3-sphere. This "real" form of $\mathrm{SL}_{2}(\mathbf{C})$ - often known as $\mathrm{SU}_{2}(\mathbf{C})$ is called the compact form. The other real form- $\mathrm{SL}_{2}(\mathbf{R})$ - is called the split form.

This example is part of a general pattern. Each simple Lie group over the complex numbers has at least two real forms - a split form and a compact formand some have more. For example, $\mathrm{SL}_{4}(\mathbf{C})$ has three real forms: the split form $\mathrm{SL}_{4}(\mathbf{R})$, the compact form $\mathrm{SU}_{4}(\mathbf{C})$, and an "intermediate" form, $\mathrm{SL}_{2}(\mathbf{H})$ where $\mathbf{H}$ denotes the quaternions. I say "intermediate" because the compact form and the split form are extreme cases in a way that will now become apparent.

The Dynkin diagram for $\mathrm{SL}_{4}(\mathbf{C})$ is $\mathrm{A}_{3}$, and the real forms are expressed by circling nodes: all three nodes for the split form, no nodes for the compact form, and just the middle node for $\mathrm{SL}_{2}(\mathbf{H})$.

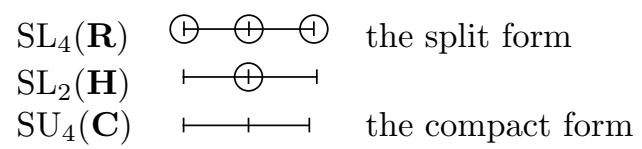

The number of circled nodes has been called by Jacques Tits the relative rank of the group. It is the same as the rank of the building for the group concerned, and the circled nodes represent the different types of vertices in this building. For example the building for $\mathrm{SL}_{4}(\mathbf{R})$ has vertices of three types: they are the 1-spaces, 2 -spaces and 3-spaces in a 4-dimensional vector space and correspond to the three nodes of the Dynkin diagram. By contrast, the building for $\mathrm{SL}_{2}(\mathbf{H})$ comprises all 1-spaces in a 2-dimensional vector space over the quaternions; these represent certain 2 -spaces in a 4 -dimensional vector space and hence belong to the middle node of the $\mathrm{A}_{3}$ diagram, the one that is circled. Finally, the building for $\mathrm{SU}_{4}(\mathbf{C})$ is vacuous, and this is true for any compact form.

Simple Lie groups over the complex numbers generalize to simple algebraic groups over an algebraically closed field, and the classification remains the same. Descending to a subfield $k$ yields $k$-forms. The split form over the real numbers has its analogue over any field, but the compact real form does not always have an analogue. When it does, the form is called anisotropic - but there is, for example, no anisotropic form of any kind over a finite field. Forty years ago, Tits [3] gave a classification of the possible $k$-forms, along with helpful comments as to the types of fields $k$ over which they could exist. Not all forms occur over the real numbers, but for those that do, at most one group is possible. This is not true in general; for example there is only one quaternion division algebra over the reals, but infinitely many over the rationals.

\section{Rank 2}

When the relative rank is 2 there are two circled nodes, and the buildings are 'Moufang polygons'. A Moufang polygon is an irreducible building of rank 2 that admits the action of root groups, and in a major book on the topic, Tits and Weiss 
[8] classify them all. An earlier theorem, proved independently by Tits and Weiss [5], [9], showed that a Moufang polygon must be a generalized 3-gon, 4-gon, 6-gon, or 8-gon, so the purpose of [8] was to analyze these four cases. As an example I want to briefly summarize the classification of Moufang 3-gons - these are projective planes, the points and lines of the plane being the vertices of the building.

To illustrate the main types of Moufang 3-gons it suffices to work with real forms of Lie groups. In this case the projective plane has a coordinate ring that is a real division algebra, and there are four of these, having dimensions 1, 2, 4, and 8: the real numbers, the complex numbers, the quaternions, and the octonions.

\begin{tabular}{ccc}
\hline Coordinatizing ring & Simple Lie group & \multicolumn{2}{c}{ Diagram } \\
\hline $\begin{array}{c}\text { real or complex numbers } \\
\text { quaternions }\end{array}$ & $\mathrm{SL}_{3}(\mathbf{R})$ or $\mathrm{SL}_{3}(\mathbf{C})$ & $\mathrm{SL}_{3}(\mathbf{H})$ - a real form of $\mathrm{A}_{5}$ \\
octonions & a real form of $\mathrm{E}_{6}$
\end{tabular}

In the first diagram the circled nodes represent 1-spaces and 2-spaces in a threedimensional vector space. In the second diagram the circled nodes represent 1spaces and 2-spaces in a 3-dimensional vector space over the quaternions, which in turn represent certain 2 -spaces and 4 -spaces in a 6 -dimensional complex vector space, as expressed by the circled nodes in the $\mathrm{A}_{5}$ diagram. The fourth case - a form of $\mathrm{E}_{6}$-is exceptional, and its analogue for Moufang 4-gons is a major feature of Weiss's book.

Going from the real numbers to an arbitrary field, Moufang 3-gons can be divided into three cases as above. The split case in the first diagram exists over any field. The second case extends to all associative, non-commutative division algebras; over the reals these are limited to the algebra of quaternions, which has degree 2 (and dimension 4), but some fields admit central division algebras of other degrees. The third case involves 'alternative' division algebras (which satisfy a weakened form of the associative law), and a theorem of Bruck and Kleinfeld [1], [2] shows that these are Cayley-Dickson algebras. This concludes the discussion of Moufang 3-gons.

Moufang 8-gons were classified by Tits in [7], where he showed they all arise from Lie groups of type $\mathrm{F}_{4}$. A classification of Moufang 6-gons was stated by Tits in [4], though the details remained unproven until the joint work with Weiss on Moufang Polygons [8]. The classification of Moufang 4-gons was the hardest of all, and when Tits and Weiss started to write it up, a hitherto unnoticed type came into being, arising from groups of type $\mathrm{F}_{4}$.

\section{Quadrangular algebras}

Turning to this classification and the term quadrangular algebra, one should be aware that Moufang 4-gons are also called Moufang quadrangles. They can be divided into three classes:

(i) Those arising from classical groups.

(ii) Those arising from "mixed groups" (in which there are two imperfect fields of characteristic $2, K$ and $L$, with $\left.K^{2} \subset L \subset K\right)$.

(iii) Those arising from quadrangular algebras.

There is some overlap here, in the sense that some classical groups arising from pseudo-quadratic spaces can be obtained from quadrangular algebras (which Weiss calls special), but I shall concentrate on the other, non-special ones. The most 
important of these arise from algebraic groups of types $\mathrm{E}_{6}, \mathrm{E}_{7}$, and $\mathrm{E}_{8}$. They are $k$-forms of algebraic groups belonging to the following diagrams:

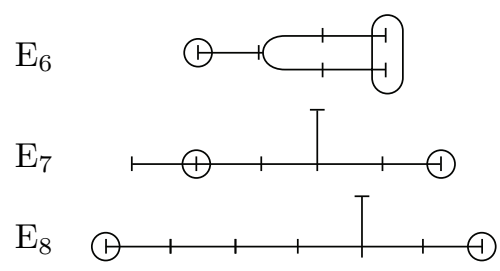

The $\mathrm{E}_{6}$ one exists over the real numbers, though the $\mathrm{E}_{7}$ and $\mathrm{E}_{8}$ ones do not. The quadrangular algebras in all these cases Weiss calls regular (but not special). There is a further type that he calls defective arising from groups of type $\mathrm{F}_{4}$. These are the most exotic of all-they involve purely inseparable field extensions in characteristic 2-and Weiss only discovered them during the joint work with Tits on the classification of Moufang 4-gons by investigating a strange lacuna that shouldn't have existed but did.

The classification of Moufang 4-gons by Tits and Weiss is related to this intriguing monograph in two ways. One is that the use of quadrangular algebras short-cuts some of the methods in [8]. The other is that the concept is an analogue to the octonion algebras, and quadratic Jordan division algebras of degree 3 , that give rise to Moufang 3-gons and 6-gons. In fact all the exceptional Moufang planes, quadrangles, and hexagons that do not arise from "mixed groups" (of characteristic 2 for quadrangles or characteristic 3 for hexagons) come from octonions, quadrangular algebras, or Jordan algebras.

A potential use for quadrangular algebras is to analyze two open questions. One is the Kneser-Tits conjecture [6] that concerns the full group of 'linear transformations' of a building (e.g. GL ${ }_{n}$ ) factored out by the subgroup generated by root groups (e.g. $\left.\mathrm{SL}_{n}\right)$. The conjecture is proved for all Moufang buildings except the 6-gons and 4-gons of type $\mathrm{E}_{8}$, in which case the group of 'linear transformations' is conjectured to be equal to the subgroup generated by root groups. For the $\mathrm{E}_{8}$ hexagons this can be rephrased as a question on quadratic Jordan algebras, and for the $\mathrm{E}_{8}$ quadrangles it can now be rephrased in terms of quadrangular algebras. Another open question about the $\mathrm{E}_{8}$ quadrangle concerns fields that are complete with respect to a discrete valuation: is there, in such cases, an affine building that yields the quadrangle as its structure 'at infinity'?

In conclusion, there have been many results on finite generalized quadrangles that fill a well-needed gap in the mathematical literature, but this monograph is much deeper and will enable progress to be made in a difficult technical area where some exotic forms of algebraic groups have hitherto been little understood.

\section{REFERENCES}

[1] R. H. Bruck and E. Kleinfeld, The structure of alternative division rings, Proc. Amer. Math. Soc. 2 (1951) 878-890. MR0045099 (13:526e)

[2] E. Kleinfeld, Alternative division rings of characteristic 2, Proc. Nat. Acad. Sci. U.S. 37 (1951) 818-820. MR0045100 (13:527a)

[3] J. Tits, Classification of algebraic semisimple groups, in Algebraic Groups and Discontinuous Subgroups, Boulder, 1965, Proc. Symp. Pure Math. 9, Amer. Math. Soc., 1966, pp. 33-62. MR0224710 (37:309) 
[4] J. Tits, Classification of buildings of spherical type and Moufang polygons: a survey, in Coll. Internaz. Teorie Combinatorie, Atti dei Convegni Lincei 17, Rome 1976, pp. 229-246. MR0444793 (56:3140)

[5] J. Tits, Non-existence de certains polygones généralisés, I, II, Inventiones Math. 36 (1976) 275-284; 51 (1979) 267-269. MR0435248 (55:8208), MR0530633 (83c:20055)

[6] J. Tits, Groupes de Whitehead de groupes algébriques simples sur un corps, Seminaire Bourbaki, 1976-77, no. 505; Lecture Notes in Mathematics 677, Springer-Verlag, 1977.

[7] J. Tits, Moufang octagons and Ree groups of type ${ }^{2} F_{4}$, Amer J. Math. 105 (1983) 539-594. MR701569 (84m:20048)

[8] J. Tits and R. Weiss, Moufang Polygons, Springer-Verlag, 2002. MR1938841 (2003m:51008)

[9] R. Weiss, The nonexistence of certain Moufang polygons, Inventiones Math. 51 (1979) 261266. MR530632 (82k:05062)

Mark A. Ronan

University of ILLINOIS AT CHICAgo E-mail address: ronan@uic.edu 